\title{
MiR-30a inhibits BECN1-mediated autophagy in diabetic cataract
}

\author{
Lu Zhang ${ }^{1,2}$, Rong Cheng ${ }^{2,3}$ and Yusen Huang ${ }^{2}$ \\ ${ }^{1}$ Department of Ophthalmology, School of Medicine, Shandong University, Jinan 250012, China \\ ${ }^{2}$ Qingdao Eye Hospital, Shandong Eye Institute, Shandong Academy of Medical Sciences, Qingdao 266071, China \\ ${ }^{3}$ College of Medicine, Qingdao University, Qingdao 266071, China \\ Correspondence to: Yusen Huang, email: huang_yusen@126.com \\ Keywords: miR-30a, BECN1, autophagy, diabetic cataract, lens epithelial cells \\ Received: May 07, $2017 \quad$ Accepted: June 25, $2017 \quad$ Published: August 24, 2017 \\ Copyright: Zhang et al. This is an open-access article distributed under the terms of the Creative Commons Attribution License 3.0 \\ (CC BY 3.0), which permits unrestricted use, distribution, and reproduction in any medium, provided the original author and source \\ are credited.
}

\section{ABSTRACT}

\begin{abstract}
Purpose: To investigate the role of microRNAs in the regulation of autophagy and apoptosis in lens epithelial cells (LECs) during diabetic cataract formation.

Methods: A miRNA microarray study and quantitative real-time PCR were performed to identify the expression of miRNAs in LECs of diabetic cataract. Human LECs were cultured in high glucose conditions as a diabetic cataract model. BECN1 and LC3B were detected by Western blotting and quantitative real-time PCR. The extent of apoptosis was measured using FACSCalibur flow cytometry.

Results: Downregulation of miR-30a was identified in LECs attached to diabetic cataract tissues. By the bioinformatic assay and the luciferase activity assay, BECN1 was found to be a direct target of miR-30a. MiR-30a reduced the BECN1-mediated autophagy activity induced by high glucose in LECs in vitro. The ratio of LECs apoptosis was also decreased.

Conclusion: MiR-30a was involved in the inhibition of autophagy by targeting BECN1 in LECs in human diabetic cataract.
\end{abstract}

\section{INTRODUCTION}

Cataracts are a leading cause of blindness and visual impairment in the world [1]. A significant relationship has been revealed between diabetes mellitus (DM) and diabetic cataract as the younger ages and severity of cataracts were found in diabetic patients [2]. One of the reasons may be the high glucose (HG) in such patients. Human lens epithelial cells (LECs) are a single layer of polygonal cuboidal cells that act as the major source of transport, metabolism and detoxification in lens development [3]. The integrity and survival of LECs are critical for lens transparency [4]. However, the molecular mechanism during the process of diabetic cataract formation remains largely unknown.

Macroautophagy (later called autophagy), a multistep process involving cellular protein and organelle degradation and nutrition recycle, is essential for cell survival and development [5-7]. It plays pathophysiologic roles in many types of cataracts, such as hereditary cataract $[8,9]$, posterior capsule opacification $[10]$ and age-related cataract [11]. The degradation and clearance of LECs protein aggregation and organelles through an autophagosomal-lysosomal pathway is critically important for maintaining lens transparency.

MicroRNAs (miRNAs) are a group of small RNAs, approximately 21-23 nucleotides in length. They have been identified as non-coding protein and silence gene expression as either mRNA degradation or translational inhibition by targeting the 3'UTR region of mRNA molecules $[12,13]$. MiRNAs have been shown to be associated with development, differentiation, maturation, proliferation, apoptosis and autophagy [13-15]. Recently, the function of miRNAs in multiple eye tissues has been disclosed. For example, miRNA-96 was observed to affect the survival and apoptosis of retinal ganglion cells through the activation of caspase-2 [16] and miRNA-100 via the phosphorylation pathway [17]. MiR-182 could 


\begin{tabular}{lcccc}
\hline & \multicolumn{4}{c}{ Mean \pm SD } \\
\cline { 2 - 5 } & Age at surgery (year) & Duration of DM (year) & Blood glucose & HbA1c (\%) \\
\hline Group 1 & $60.67 \pm 9.07$ & $9.00 \pm 3.61$ & $9.47 \pm 2.05$ & $7.77 \pm 1.40$ \\
Group 2 & $61.89 \pm 5.37$ & $10.22 \pm 6.08$ & $9.11 \pm 2.59$ & $8.33 \pm 1.51$ \\
\hline
\end{tabular}

Group 1: patients cataract tissues used for the miRNA microarray study; Group 2: patients cataract tissues used for the quantitative RT-PCR; DM: diabetes mellitus; HbA1c: glycated hemoglobin.

target NOX4 directly, thus enhancing neurite outgrowth in isolated trigeminal sensory neurons and recover corneal sensation in hyperglycemic conditions [18]. We previously found that miR-204 inhibited epithelial-mesenchymal transition (EMT) by directly targeting SMAD4 in human posterior capsule opacification [19]. In the present study, we investigated the role of miRNAs in the regulation of autophagy and apoptosis in LECs during diabetic cataract formation.

\section{RESULTS}

\section{MiR-30a was downregulated in human diabetic cataract}

To test the expression of miRNAs in LECs, three donated normal transparent lens tissues and three diabetic cataract tissues were used to perform a miRNA microarray study. The characteristics of the diabetic patients are presented in Table 1 . The patient age was $60.67 \pm 9.07$ years $($ mean \pm SD). The duration of $\mathrm{DM}$ was $9.00 \pm 3.61$ years. The blood glucose was $9.47 \pm 2.05$ $\mathrm{mmol} / \mathrm{L}$, and HbA1c (\%) was $7.77 \pm 1.40$. Ninety-two miRNAs were identified to be upregulated more than two folds in diabetic cataract tissues compared with transparent lens tissues (Figure 1A). Meanwhile, 75 miRNAs were identified to be downregulated more than two folds in diabetic cataract tissues compared with transparent lens tissues. The top 5 upregulated miRNAs were miR-431-5p, miR-3169, miR-371a-3p, miR-1537 and miR-593-3p, and the top 5 downregulated miRNAs were miR-30a-5p, miR-193a-3p, miR-204-5p, miR-184 and miR-29b-3p (Figure 1B). As miR-30a is relevant to autophagy [20] and was downregulated more than 180 folds in our miRNA expression profile, we chose it for a further investigation. A quantitative real-time PCR (RTPCR) was performed to validate the miRNA microarray study and observe the expression of miR-30a. The involved patients, aged $61.89 \pm 5.37$ years, had a duration of DM of $10.22 \pm 6.08$ years. The blood glucose was $9.11 \pm 2.59 \mathrm{mmol} / \mathrm{L}$, and $\mathrm{HbA} 1 \mathrm{c}(\%)$ was $8.33 \pm 1.51$. The expression of miR-30a was downregulated significantly in diabetic cataract tissues compared to transparent lens tissues, which corresponded to the result of the miRNA microarray study.

\section{MiR-30a directly targeted BECN1}

Lens slit lamp photos are presented in Figure 2A. Transmission electron microscopy was performed, and double membrane autophagosomes were detectable not only in LECs attached to normal tissues but also those in diabetic cataract tissues (Figure 2B). However, the single autophagosome in LECs of diabetic cataract tissues was larger than that in normal LECs. Moreover, multiple mitochondria were encapsulated in one autophagosome in LECs attached to diabetic cataract tissues, which illustrated the inverted autophagy activity induced by high blood glucose in patients with diabetes.

From a bioinformatics database (TargetScanHuman, http://www.targetscan.org/vert 70/) we found that miR30a could target BECN1 3'UTR, an important regulator of autophagy, at positions of 240-247 (Figure 2C). MiR30a agomir could overexpress miR-30a, while miR-30a antagomir could inhibit the expression of miR-30a. A dual luciferase reporter assay was performed to identify the direct relationship of miR-30a and BECN1. The relative luciferase activity was observed to decrease significantly when the transfected miR-30a agomir and the 3'UTR mutant of BECN1 abolished the suppressive effect of miR-30a on BECN1 (Figure 2D). Western blotting was also performed, and the protein expression of BECN1 was found to be decreased by miR-30a agomir in LECs in the presence of $\mathrm{HG}$ and be increased by miR-30a antagomir when compared with the negative control (NC) (Figure $2 \mathrm{E}$ ). The mRNA expression was not affected by miR$30 \mathrm{a}$ agomir or antagomir (data not shown). All the results revealed the repression of miR-30a on BECN1 at the translational level. Because BECN1 is a key regulator of autophagy, we next investigated the regulation of miR-30a on BECN1-mediated autophagy activity in LECs.

\section{MiR-30a repressed autophagy in LECs}

LECs were exposed to $5 \mathrm{mM}$ D-glucose (normal glucose) as a control group and $30 \mathrm{mM}$ D-glucose (HG) as an experiment group, respectively, for 48 hours, and then treated with miR-30a agomir and agomir NC directly for 24 hours. In the $\mathrm{HG}$ environment, the ratio of LC3B II and LC3B I proteins increased significantly in LECs; the expression was decreased when LECs were transfected 
with miR-30a agomir compared with agomir NC (Figure 3A). As LC3B II was measured as an autophagosomeassociated marker [21], the increased ratio of LC3B II/ LC3B I represented the active autophagy activity. We also employed BECN1 siRNA to knock down the gene endogenous expression and found that BECN1 siRNA decreased the ratio of LC3B II/LC3B I. The increased LC3B mRNA expression was induced by $\mathrm{HG}$, and miR30a agomir inhibited the HG-induced LC3B expression (Figure 3B). The above findings indicated that $\mathrm{HG}$

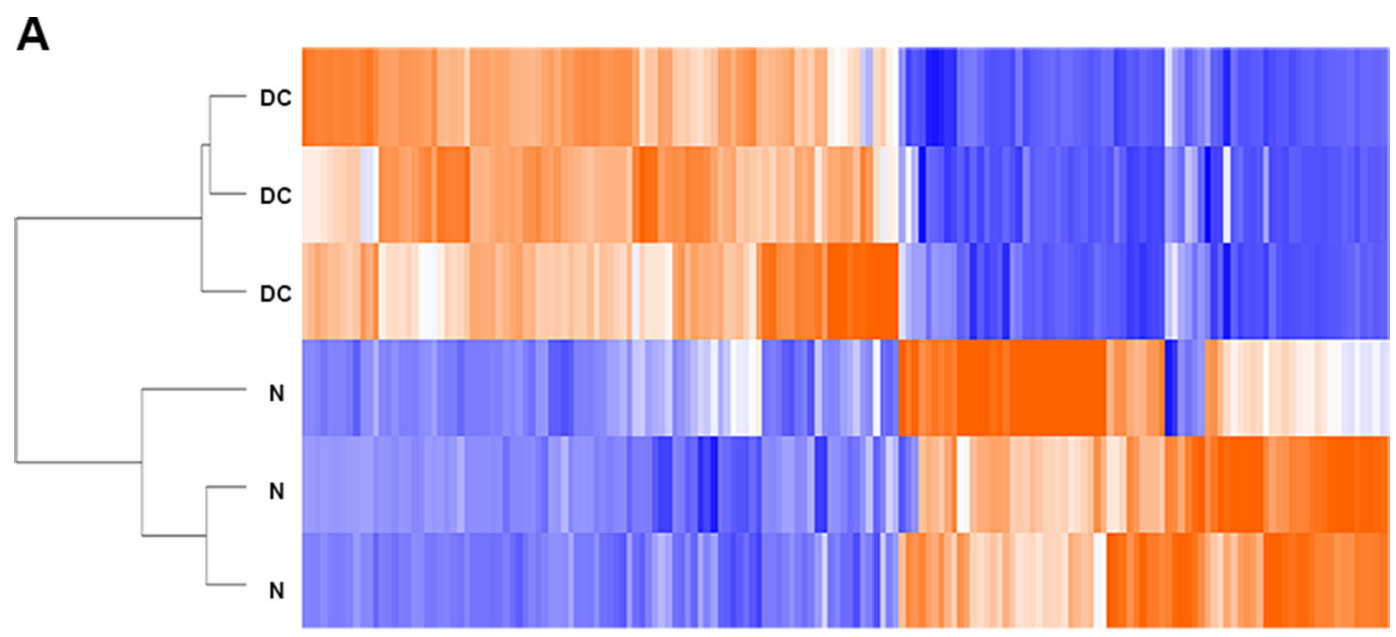

B
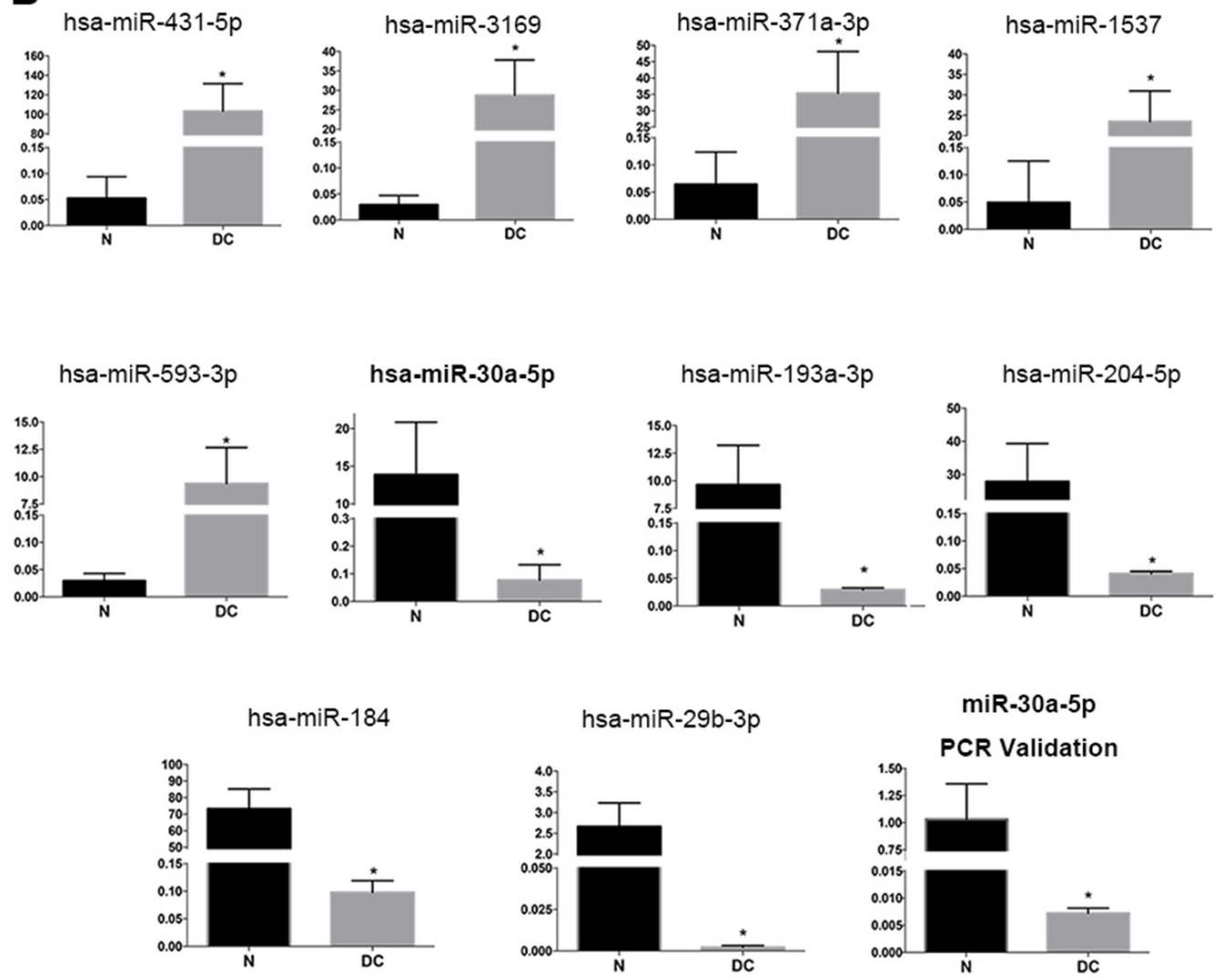

Figure 1: Expression of miRNAs in human diabetic cataract tissues. (A) The heatmap of three normal lens samples (N) and three diabetic cataract samples (DC). A total of 92 miRNAs were identified to be upregulated more than two folds in diabetic cataract tissues, and 75 miRNAs were identified to be downregulated. (B) The expression of the top 5 upregulated miRNAs (miR-431-5p, miR3169, miR-371a-3p, miR-1537 and miR-593-3p) and top 5 downregulated miRNAs (miR-30a-5p, miR-193a-3p, miR-204-5p, miR-184 and miR-29b-3p) (*P<0.005, N as control). Real-time PCR was performed using nine normal lens tissues and nine diabetic cataract tissues. The relative expression levels of hsa-miR-30a were downregulated significantly in lens epithelial cells attached to diabetic cataract compared with those in normal lens tissues $(* \mathrm{P}<0.05, \mathrm{~N}$ as control). Significant differences are indicated by t-test $(* \mathrm{P}<0.05)$. 
stimulated the autophagy activity in LECs, and miR-30a repressed the level of autophagy by modulating BECN1 expression.

\section{MiR-30a decreased apoptosis of LECs in an HG condition}

As both autophagy and apoptosis are involved in cell death, we tried to investigate whether miR-30a could regulate the LECs apoptosis. In apoptotic cells, FITC could conjugate annexin $\mathrm{V}$ and invert phosphatidylserine. By flow cytometry, we observed that LECs apoptosis was induced by HG (Figure 4). The rate of HG-induced apoptosis in LECs increased from $4.94 \%$ to $27.93 \%$. Meanwhile, the rate of LECs apoptosis decreased from $16.42 \%$ to $9.8 \%$ when LECs were treated with miR-30a agomir compared to agomir NC. These results revealed that miR-30a might help protect LECs from apoptosis in an $\mathrm{HG}$ condition.

\section{DISCUSSION}

Since the function of miRNAs in LECs during diabetic cataract formation was not well unknown, we performed a miRNA microarray study using normal transparent lens tissues and diabetic cataract tissues. MiR-30a was chosen for a further study because it was reported to be related with autophagy, an intracellular self-defense mechanism for sustaining of metabolic homoeostasis [22-24]. There were some reports on diabetic cataract using $\mathrm{HG}$ conditions in vitro [25-27]. Du et al.[27] set $50 \mathrm{mmol} / \mathrm{L}$ glucose as HG conditions in treating rat lenses, finding that all the lenses in the normal glucose group $(5.56 \mathrm{mmol} / \mathrm{L}$ glucose $)$
A
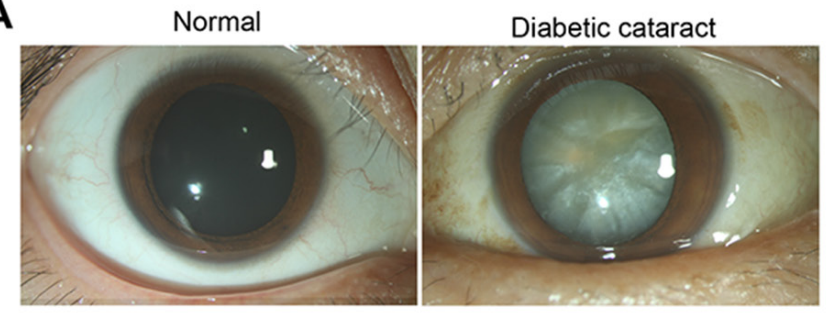

C

Position 240-247 of BECN1 3' UTR 5'. AUAUUAAACAGUACAUGUUUACA hsa-miR-30a $3^{\prime}$ GAAGGUCAGCUCCUACAAAUGU

D

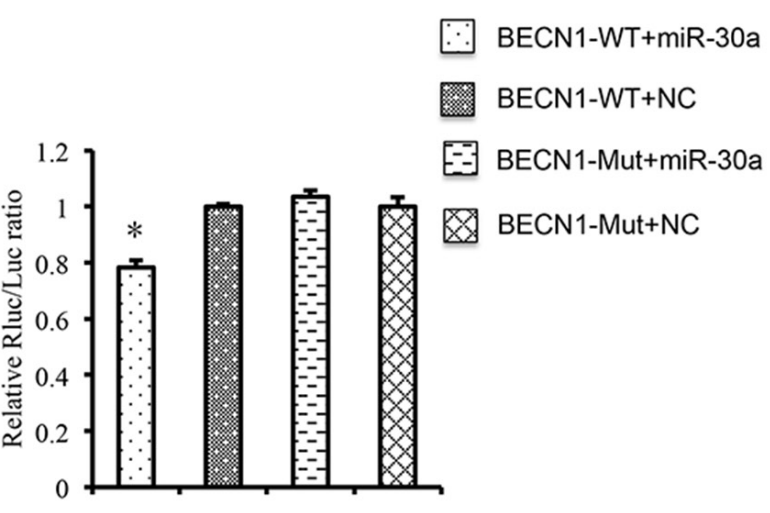

B

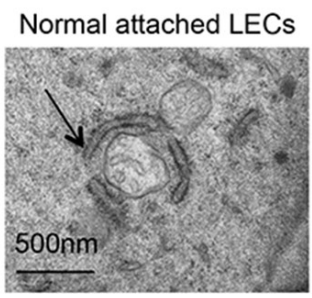

DC attached LECs

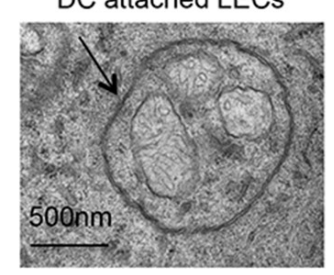

E
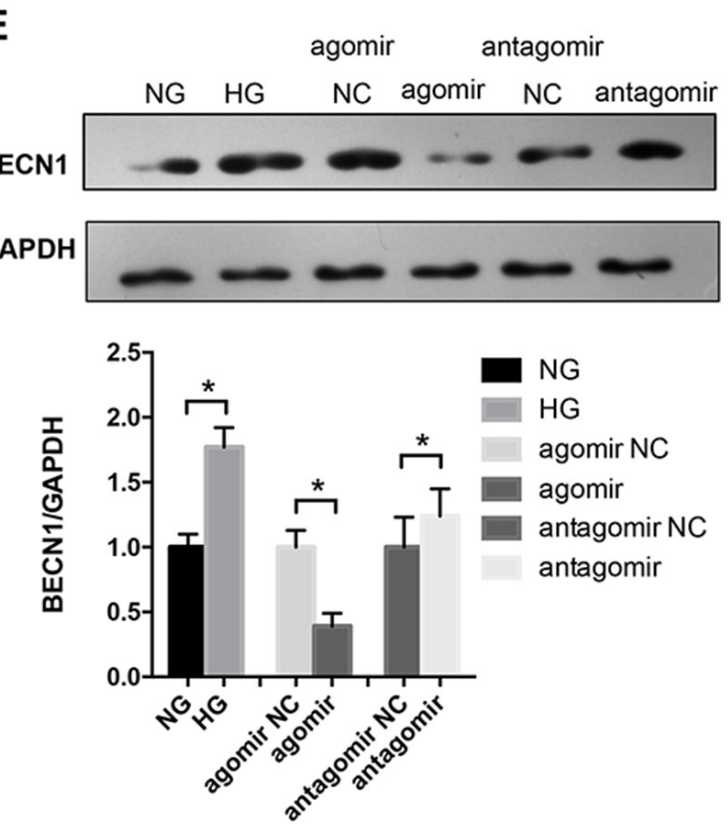

Figure 2: MiR-30a targeted human BECN1 and downregulated BECN1 protein. (A) Slit lamp photos of a transparent lens vs. an opaque lens in a patient with diabetic cataract. (B) Electron micrographs of double membrane autophagosomes in lens epithelial cells (LECs). The autophagosomes encapsulated several mitochondria in LECs of diabetic cataract and were larger than those normal ones. (C) Position of the miR-30a targeted sequence in the 3'-UTR of BECN1 mRNA. (D) In a dual luciferase reporter assay, the relative luciferase activity decreased significantly when pmiR-RB-REPORT-BECN1-3'UTR and miR-30a agomir were cotransfected (agomir NC as control, $\left.{ }^{*} \mathrm{P}<0.05\right)$. The mutation of the perfectly complementary sites in the BECN1-3'UTR abolished the suppressive effect of miR-30a on BECN1. (E) Western blot analysis. High glucose induced the protein expression of BECN1 in LECs $(* \mathrm{P}<0.05$, NG as control). BECN1 expression was downregulated when LECs were transfected with miR-30a agomir and upregulated with miR-30a antagomir $(* \mathrm{P}<0.05$, agomir $\mathrm{NC}$ and antagomir NC as control). Significant differences are indicated by t-test $(* \mathrm{P}<0.05)$. 
appeared clear while most lenses in the HG group were covered with opaque rings after 5 days. In our previous study [28], human lens capsular bags were maintained in $5 \mathrm{mM}$ D-glucose or $25 \mathrm{mM}$ D-glucose as a diabetic cataract model in vitro, which adequately recapitulated the diabetic state in vivo. Thus, we chose human lens epithelial cells cultured in HG conditions as the diabetic cataract model in the present study. We identified that miR-30a was downregulated in LECs attached to diabetic cataract tissues; meanwhile, miR30 a could decrease the levels of HG-induced autophagy by targeting BECN1 in LECs besides inhibiting LECs apoptosis in $\mathrm{HG}$.

miRNAs are involved in almost every aspect of cellular processes like differentiation, proliferation, apoptosis, metabolism and environmental stress [13], so
A
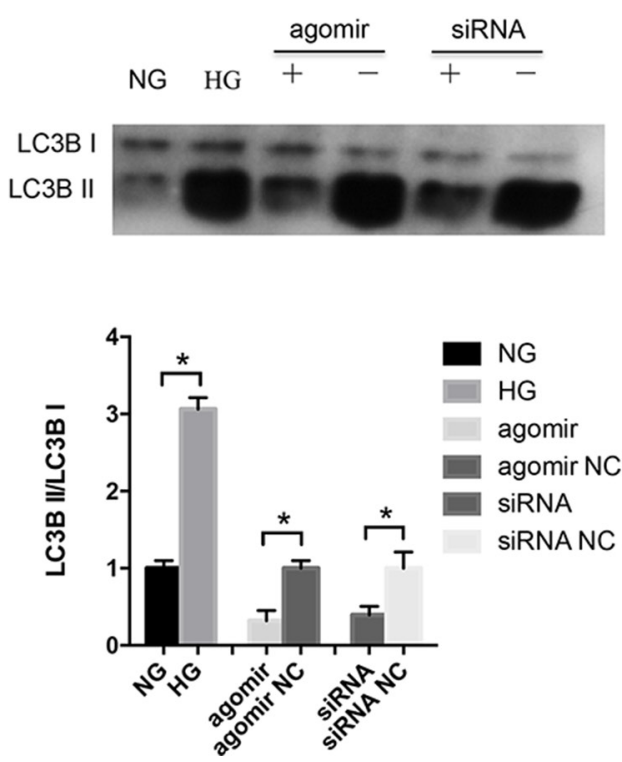

B

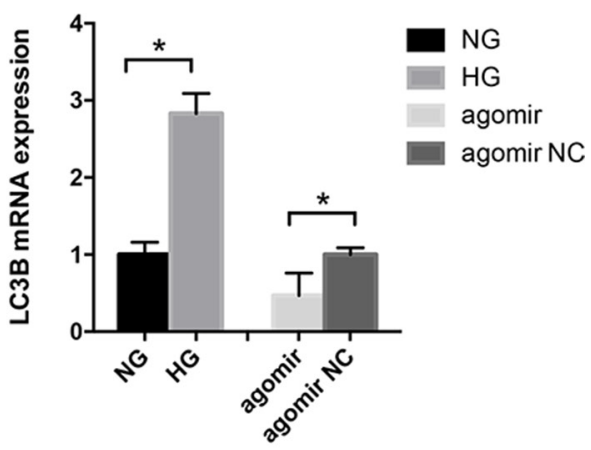

Figure 3: MiR-30a regulated autophagy in lens epithelial cells (LECs). (A) Western blot analysis. In high glucose, the ratio of LC3B II/LC3B I was elevated compared with in normal glucose $\left({ }^{*} \mathrm{P}<0.05\right)$. MiR-30a agomir downregulated the ratio directly (agomir NC as control, $\left.{ }^{*} \mathrm{P}<0.05\right)$; BECN1 siRNA also repressed LC3B II expression compared to siRNA NC $(\mathrm{P} *<0.05)$. (B) The level of LC3B mRNA expression showed an increase in LECs due to high glucose and a decrease when LECs were treated with miR-30a agomir (agomir NC as control, $\left.{ }^{*} \mathrm{P}<0.05\right)$. Significant differences are indicated by t-test $(* \mathrm{P}<0.05)$.
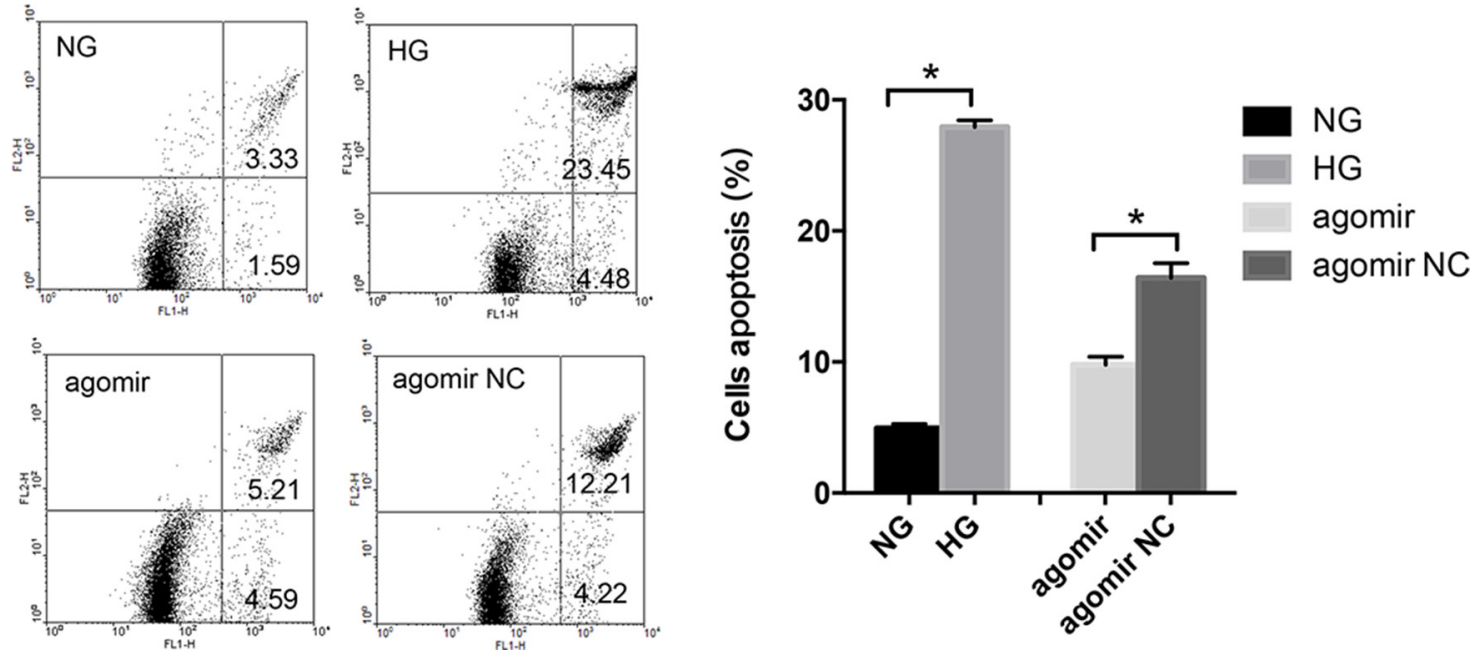

Figure 4: MiR-30a influenced high glucose-induced lens epithelial cells (LECs) apoptosis. Lower left: survivals; lower right: early apoptosis; upper right: post-apoptotic necrosis. The apoptosis rate of LECs was from $4.94 \%$ to $27.93 \%$ in the presence of high glucose $(* \mathrm{P}<0.05, \mathrm{NG}$ as control); the rate decreased from $16.42 \%$ to $49.8 \%$ when LECs were treated with miR-30a agomir $(* \mathrm{P}<0.05$, agomir $\mathrm{NC}$ as control). Significant differences are indicated by t-test $(* \mathrm{P}<0.05)$. 
many diseases have been investigated to be associated with miRNA expression [29]. miRNAs have a great relationship with the lens development and cataract formation. However, there has been no report about the effect of miR-30a on LECs during cataract formation. Studies of miR-30a have been focused mainly on diseases like cancers, ischemic injury and cardiac pathology [24, $30,31]$. In age-related cataract, miR-15a-5p, miR-15a-3p and miR-16-1-5p were reported to increase in LECs and regulate apoptosis by suppressing the expression of the anti-apoptotic genes [32]. MiR-34a was highly expressed in unclear, cortical and posterior subcapsular cataract tissues and was correlated with lens opacity severity [33]. Moreover, miR-34a could enhance the apoptosis by downregulating Bcl-2 and SIRT1 in human LECs [34]. Let- $7 \mathrm{~b}$ is correlated with age-related cataract and can enhance the ultraviolet irradiation-induced apoptosis of LECs [35]. The increase of let-7b may represent a risk factor in the formation of age-related cataracts [36]. Although miR-29a and miRNA-29c might target the BCL2-modifying factor to regulate apoptosis levels induced by $\mathrm{HG}$ in the LECs of diabetic rats [37], the contributions of miRNAs to the human diabetic cataract pathogenesis are not clearly understood.

BECN1 is involved in multiple cellular processes and is thought to be a key modulator of cellular autophagy through mediating vesicle-trafficking processes. It has been identified as a direct target of miR30 a mostly in cancers and ischemic injury $[38,39]$. In this study, we identified that HG induced the increase of BECN1 in LECs and did help miR-30a to regulate autophagy activity in LECs. By transmission electron microscopy, larger double membrane autophagosomes were shown encapsulate several mitochondria in LECs attached to diabetic cataract tissues compared with those in normal lens tissues. The ratio of LC3B II, a marker related to autophagosomes [21], and LC3B I revealed the repressing regulation of miR-30a on autophagy through BECN1. While excess autophagy has been implicated in type II cell death, apoptosis is described as a physiological cell death pathway. Apoptosis is commonly necessary in the differentiation and development [40, 41]. A few reports have demonstrated the complex interaction between autophagy and apoptosis [42-45]. Autophagy was observed to promote apoptosis via the Akt/mTOR signaling pathway in breast cancer cell lines during the combined treatment [46]. Moreover, miR-30a and miR-205 were reported to simultaneously suppress TP53INP1 expression to mediate apoptosis after irradiation [22]. In our study, miR-30a depressed LECs apoptosis induced by HG. However, the relationship of autophagy and apoptosis and the mechanism that miR30 a decreases the levels of apoptosis responding to HG in the process of diabetic cataract formation require further investigations.

\section{MATERIALS AND METHODS}

\section{Reagents and chemicals}

All miR-30a agomir, agomir $\mathrm{NC}$, antagomir, antagomir NC and BECN1 siRNA were purchased from RiboBio (Guangzhou, China). Anti-LC3B antibody was obtained from Invitrogen (Frederick, MD, USA), antiBECN1 from Abcam (Cambridge, MA, USA), and antiGAPDH from Kangchen Bio-tech (Shanghai, China).

\section{Lens tissue sample collection and cell culture}

This study was performed following the tenets of the Declaration of Helsinki and was approved by the ethics committee of Shandong Eye Institute. Diabetic cataract tissues were collected at surgery (YH). Normal lens tissues were provided by the eye bank of Shandong Eye Institute.

Human LECs-B3 were maintained in DMEM/ F12 with $10 \%$ fetal bovine serum, $1 \%$ penicillin $\mathrm{G}$ and $1 \%$ streptomycin containing $5 \mathrm{mM}$ D-glucose (normal glucose) and $30 \mathrm{mM}$ D-glucose (HG), respectively, in a humidified $5 \% \mathrm{CO}_{2}$ incubator at $37^{\circ} \mathrm{C}$. After being cultured for 48 hours, LECs were transfected with $200 \mathrm{nmol} / \mathrm{L}$ miR-30a agomir, agomir NC, antagomir and antagomir NC, respectively, for another 24 hours according to the manufacturer's protocol. BECN1 si-RNA was transfected using lipofectamine ${ }^{\mathrm{TM}} 2000$ (Invitrogen) for 48 hours. Subsequently, cells were washed twice with cold phosphate-buffered saline and collected for further analysis. The sequences of BECN1 siRNA were as follows: 5'-CAGUUUGGCACAAUCAAUA-3'.

\section{MiRNA microarray}

The miRNA microarray study was performed at Kangchen Bio-tech using a miRCURYTM LNA array (v.18.0, Exiqon, Vedbaek, Denmark). All the RNAs were isolated using TRIzol (Invitrogen) and a miRNeasy mini kit (QIAGEN, Hilden, Germany) according to the manufacturers' instructions. After RNA isolation from the samples, the miRCURY'TM $\mathrm{Hy} 3^{\mathrm{TM}} / \mathrm{Hy} 5^{\mathrm{TM}}$ Power labeling kit (Exiqon) was used following the manufacturer's guideline for miRNA labelling. Next, the Hy3TM-labeled samples were hybridized on the miRCURYTM LNA array (v.18.0, Exiqon) according to the array manual. Scanned images were then imported into GenePix Pro 6.0 software (Axon) for grid alignment and data extraction. The heatmap was performed using Cluster 3.0 and TreeView 1.1.6r4.

\section{Quantitative RT-PCR}

Total RNA was isolated with a miRNeasy mini kit (QIAGEN) according to the manufacturer's protocol. The cDNA of miR-30a and U6 were synthesized with a miRNA 
first-strand synthesis kit (Clontech, Dalian, China), and the cDNAs of BECNI and LC3B were synthesized with an MMLV first-strand synthesis kit (Invitrogen). The SYBR Green (Clontech) was used on an ABI 7500 system (Applied Biosystems, Foster City, CA, USA). GAPDH and U6 were used to normalize the expression level. All reactions were performed in triplicate. The 2 $-\Delta \Delta \mathrm{Ct}$ method was utilized to calculate the expression of each mRNA. The primer sequences employed were as follows: LC3B F'CCTTGTACCTGACCATGTCAACA, R'CCTGGGAGGCATAGACCATGTA.BECN1.

F' TGTCCACAGAAAGTGCCAACA, R' CCTCA CAGAGTGGGTGATCCA. The primers of miR-30a and U6 were purchased from Takara (Dalian, China) without sequence information.

\section{Luciferase activity assay}

The 3'UTR of BECN1 containing the putative target site for miR-30a and the mutant sequences were amplified by PCR and inserted into the pmiR-RBREPORT (RiboBio). By employing Lipofectamine 2000 (Invitrogen), the cells were transiently transfected with the wild-type or mutant reporter plasmid, miRNA agomir or agomir NC for 48 hours. Subsequently, luciferase activity was measured with the Dual-Luciferase Assay-System (Promega). All reactions were tested in triplicate.

\section{Transmission electron microscopy}

Normal human lenses and diabetic cataract tissues were fixed with $2.5 \%$ glutaraldehyde for 4 hours or longer and then with $1 \%$ osmic acid for 1 to 1.5 hours. The tissues were dehydrated in 50\%, 70\%, 90\% and 100\% acetone three times, each for 15 minutes, before embedded in epoxy resin (EMS, Epon 812, 14120). Sections of $70 \mathrm{~nm}$ in thickness were cut (Reichert-Jung ULTRACUT) and collected with a copper net. Thin sections were stained with uranyl acetate and lead citrate, each for 15 minutes, and viewed under a transmission electron microscope (JEM1200).

\section{Western blot analysis}

The samples were homogenized in buffer containing RIPA and phenylmethylsulfonyl fluoride. The homogenates were assayed on polyacrylamide gels, transferred onto PVDF membranes (Thermo Fisher Scientific, Billerica, USA) and then probed with specific primary antibodies.

\section{Assessment of apoptotic cells}

The extent of apoptosis was measured through a sannexinV-FITC apoptosis detection kit (Beyotime Institute of Biotechnology, Hangzhou, China) as described in the manufacturer's instruction. The cells were gently resuspended in annexin- $\mathrm{V}$ binding buffer and incubated with annexinV-FITC/PI in the dark for 15 minutes. The samples were analyzed using FACSCalibur flow cytometry (BD Bioscience). The fraction of cell population in different quadrants was analyzed using quadrant statistics. Cells in the lower left quadrant represented survivals, in the lower right quadrant represented early apoptosis, and in the upper right quadrant represented post-apoptotic necrosis.

\section{Statistical analysis}

All experiments were performed three times. All results are expressed as means \pm standard deviation (SD) unless indicated otherwise. The analysis of differential expression was performed using SPSS software (Version 16.0, Chicago, IL, USA) and GraphPad Prism software 5.0 (GraphPad Software). A P value of $<0.05$ was considered statistically significant.

\section{CONCLUSIONS}

Our study provides important insights into the role of miR-30a in the suppression of autophagy by targeting BECN1 in LECs and addresses the role of miR-30a in LECs in human diabetic cataract. Further studies are needed to focus on the potential development of miR-30a as a new therapeutic agent for diabetic cataract.

\section{ACKNOWLEDGMENTS}

This study was supported by the National Natural Science Foundation of China (81370996 and 81670839) and the Innovation Project of Shandong Academy of Medical Sciences.

\section{CONFLICTS OF INTEREST}

No conflicting relationship exists for any author.

\section{REFERENCES}

1. Resnikoff S, Pascolini D, Etya'ale D, Kocur I, Pararajasegaram R, Pokharel GP, Mariotti SP. Global data on visual impairment in the year 2002. Bull World Health Organ. 2004; 82: 844-51. https://doi.org/ S0042-96862004001100009.

2. Pollreisz A, Schmidt-Erfurth U. Diabetic cataractpathogenesis, epidemiology and treatment. J Ophthalmol. 2010; 2010: 608751. https://doi.org/10.1155/2010/608751.

3. Andley UP. The lens epithelium: focus on the expression and function of the alpha-crystallin chaperones. Int 
J Biochem Cell Biol. 2008; 40: 317-23. https://doi. org/10.1016/j.biocel.2007.10.034.

4. Sharma KK, Santhoshkumar P. Lens aging: effects of crystallins. Biochim Biophys Acta. 2009; 1790: 1095-108. https://doi.org/10.1016/j.bbagen.2009.05.008.

5. Yang Z, Klionsky DJ. Eaten alive: a history of macroautophagy. Nat Cell Biol. 2010; 12: 814-22. https:// doi.org/10.1038/ncb0910-814.

6. Rubinsztein DC. The roles of intracellular proteindegradation pathways in neurodegeneration. Nature. 2006; 443: 780-6. https://doi.org/10.1038/nature05291.

7. Williams A, Jahreiss L, Sarkar S, Saiki S, Menzies FM, Ravikumar B, Rubinsztein DC. Aggregate-prone proteins are cleared from the cytosol by autophagy: therapeutic implications. Curr Top Dev Biol. 2006; 76: 89-101. https:// doi.org/10.1016/S0070-2153(06)76003-3.

8. Andley UP, Goldman JW. Autophagy and UPR in alphacrystallin mutant knock-in mouse models of hereditary cataracts. Biochim Biophys Acta. 2016; 1860: 234-9. https://doi.org/10.1016/j.bbagen.2015.06.001.

9. Wignes JA, Goldman JW, Weihl CC, Bartley MG, Andley UP. p62 expression and autophagy in alphaB-crystallin R120G mutant knock-in mouse model of hereditary cataract. Exp Eye Res. 2013; 115: 263-73. https://doi. org/10.1016/j.exer.2013.06.026.

10. Chandler HL, Gervais KJ, Lutz EA, Curto EM, Matusow RB, Wilkie DA, Gemensky-Metzler AJ. Cyclosporine A prevents ex vivo PCO formation through induction of autophagy-mediated cell death. Exp Eye Res. 2015; 134: 63-72. https://doi.org/10.1016/j.exer.2015.03.020.

11. Morishita H, Eguchi S, Kimura H, Sasaki J, Sakamaki Y, Robinson ML, Sasaki T, Mizushima N. Deletion of autophagy-related 5 (Atg5) and Pik3c3 genes in the lens causes cataract independent of programmed organelle degradation. J Biol Chem. 2013; 288: 11436-47. https://doi. org/10.1074/jbc.M112.437103.

12. Ambros V. The functions of animal microRNAs. Nature. 2004; 431: 350-5. https://doi.org/10.1038/nature02871.

13. Bartel DP. MicroRNAs: genomics, biogenesis, mechanism, and function. Cell. 2004; 116: 281-97.

14. Zhang B, Wang Q, Pan X. MicroRNAs and their regulatory roles in animals and plants. J Cell Physiol. 2007; 210: 27989. https://doi.org/10.1002/jcp.20869.

15. Qin Y, Zhao J, Min X, Wang M, Luo W, Wu D, Yan Q, Li J, Wu X, Zhang J. MicroRNA-125b inhibits lens epithelial cell apoptosis by targeting p53 in age-related cataract. Biochim Biophys Acta. 2014; 1842: 2439-47. https://doi. org/10.1016/j.bbadis.2014.10.002.

16. Wang S, Li K. MicroRNA-96 regulates RGC-5 cell growth through caspase-dependent apoptosis. Int J Clin Exp Med. 2014; 7: 3694-702.

17. Kong N, Lu X, Li B. Downregulation of microRNA-100 protects apoptosis and promotes neuronal growth in retinal ganglion cells. BMC Mol Biol. 2014; 15: 25. https://doi. org/10.1186/s12867-014-0025-1.

18. Wang Y, Zhao X, Wu X, Dai Y, Chen P, Xie L. microRNA-182 mediates sirt1-induced diabetic corneal nerve regeneration. Diabetes. 2016; 65: 2020-31. https:// doi.org/10.2337/db15-1283.

19. Wang Y, Li W, Zang X, Chen N, Liu T, Tsonis PA, Huang Y. MicroRNA-204-5p regulates epithelial-to-mesenchymal transition during human posterior capsule opacification by targeting SMAD4. Invest Ophthalmol Vis Sci. 2013; 54: 323-32. https://doi.org/10.1167/iovs.12-10904.

20. Zhu H, Wu H, Liu X, Li B, Chen Y, Ren X, Liu CG, Yang JM. Regulation of autophagy by a beclin 1-targeted microRNA, miR-30a, in cancer cells. Autophagy. 2009; 5: 816-23.

21. Klionsky DJ, Abdelmohsen $\mathrm{K}$, Abe A, Abedin MJ, Abeliovich H, Acevedo Arozena A, Adachi H, Adams CM, Adams PD, Adeli K, Adhihetty PJ, Adler SG, Agam G, et al. Guidelines for the use and interpretation of assays for monitoring autophagy (3rd edition). Autophagy. 2016; 12: 1-222. https://doi.org/10.1080/15548627.2015.1100356.

22. Xu CG, Yang MF, Fan JX, Wang W. MiR-30a and miR-205 are downregulated in hypoxia and modulate radiosensitivity of prostate cancer cells by inhibiting autophagy via TP53INP1. Eur Rev Med Pharmacol Sci. 2016; 20: 1501-8.

23. Zheng B, Zhu H, Gu D, Pan X, Qian L, Xue B, Yang D, Zhou J, Shan Y. MiRNA-30a-mediated autophagy inhibition sensitizes renal cell carcinoma cells to sorafenib. Biochem Biophys Res Commun. 2015; 459: 234-9. https://doi. org/10.1016/j.bbrc.2015.02.084.

24. Wang P, Zhang N, Liang J, Li J, Han S, Li J. Micro-RNA$30 \mathrm{a}$ regulates ischemia-induced cell death by targeting heat shock protein HSPA5 in primary cultured cortical neurons and mouse brain after stroke. J Neurosci Res. 2015; 93: 1756-68. https://doi.org/10.1002/jnr.23637.

25. Olofsson EM, Marklund SL, Karlsson K, Brannstrom T, Behndig A. In vitro glucose-induced cataract in copper-zinc superoxide dismutase null mice. Exp Eye Res. 2005; 81: 639-46. https://doi.org/10.1016/j.exer.2005.03.022.

26. Olofsson EM, Marklund SL, Behndig A. Glucose-induced cataract in CuZn-SOD null lenses: an effect of nitric oxide? Free Radic Biol Med. 2007; 42: 1098-105. https://doi. org/10.1016/j.freeradbiomed.2007.01.012.

27. Du L, Hao M, Li C, Wu W, Wang W, Ma Z, Yang T, Zhang N, Isaac AT, Zhu X, Sun Y, Lu Q, Yin X. Quercetin inhibited epithelial mesenchymal transition in diabetic rats, high-glucose-cultured lens, and SRA01/04 cells through transforming growth factor-beta2/phosphoinositide 3-kinase/Akt pathway. Mol Cell Endocrinol. 2017; 452: 44-56. https://doi.org/10.1016/j.mce.2017.05.011.

28. Zhang L, Wang Y, Li W, Tsonis PA, Li Z, Xie L, Huang Y. MicroRNA-30a regulation of epithelial-mesenchymal transition in diabetic Cataracts Through Targeting 
SNAI1. Sci Rep. 2017; 7: 1117. https://doi.org/10.1038/ s41598-017-01320-3.

29. Harries LW. MicroRNAs as mediators of the ageing process. Genes (Basel). 2014; 5: 656-70. https://doi.org/10.3390/ genes5030656.

30. Yang Y, Li Y, Chen X, Cheng X, Liao Y, Yu X. Exosomal transfer of miR-30a between cardiomyocytes regulates autophagy after hypoxia. J Mol Med (Berl). 2016; 94: 71124. https://doi.org/10.1007/s00109-016-1387-2.

31. Cheng Y, Chen G, Hu M, Huang J, Li B, Zhou L, Hong L. Has-miR-30a regulates autophagic activity in cervical cancer upon hydroxycamptothecin exposure. Biomed Pharmacother. 2015; 75: 67-74. https://doi.org/10.1016/j. biopha.2015.08.034.

32. Li Y, Liu S, Zhang F, Jiang P, Wu X, Liang Y. Expression of the microRNAs hsa-miR-15a and hsa-miR-16-1 in lens epithelial cells of patients with age-related cataract. Int J Clin Exp Med. 2015; 8: 2405-10.

33. Chien KH, Chen SJ, Liu JH, Chang HM, Woung LC, Liang CM, Chen JT, Lin TJ, Chiou SH, Peng CH. Correlation between microRNA-34a levels and lens opacity severity in age-related cataracts. Eye (Lond). 2013; 27: 883-8. https:// doi.org/10.1038/eye.2013.90.

34. Li QL, Zhang HY, Qin YJ, Meng QL, Yao XL, Guo HK. MicroRNA-34a promoting apoptosis of human lens epithelial cells through down-regulation of B-cell lymphoma-2 and silent information regulator. Int $\mathrm{J}$ Ophthalmol. 2016; 9: 1555-60. https://doi.org/10.18240/ ijo.2016.11.04.

35. Dong Y, Zheng Y, Xiao J, Zhu C, Zhao M. MicroRNA let-7b induces lens epithelial cell apoptosis by targeting leucine-rich repeat containing $\mathrm{G}$ protein-coupled receptor 4 (Lgr4) in age-related cataract. Exp Eye Res. 2016; 147: 98-104. https://doi.org/10.1016/j.exer.2016.04.018.

36. Peng CH, Liu JH, Woung LC, Lin TJ, Chiou SH, Tseng PC, Du WY, Cheng CK, Hu CC, Chien KH, Chen SJ. MicroRNAs and cataracts: correlation among let-7 expression, age and the severity of lens opacity. $\mathrm{Br} \mathrm{J}$ Ophthalmol. 2012; 96: 747-51. https://doi.org/10.1136/ bjophthalmol-2011-300585.

37. Sun Y, Lu CM, Song Z, Xu KK, Wu SB, Li ZJ. Expression and regulation of microRNA-29a and microRNA-29c in early diabetic rat cataract formation. Int $\mathrm{J}$ Ophthalmol. 2016; 9: 1719-24. https://doi.org/10.18240/ijo.2016.12.03.
38. Zou Z, Wu L, Ding H, Wang Y, Zhang Y, Chen X, Chen X, Zhang CY, Zhang Q, Zen K. MicroRNA-30a sensitizes tumor cells to cis-platinum via suppressing beclin 1-mediated autophagy. J Biol Chem. 2012; 287: 4148-56. https://doi.org/10.1074/jbc.M111.307405.

39. Wang P, Liang J, Li Y, Li J, Yang X, Zhang X, Han S, Li $\mathrm{S}$, Li J. Down-regulation of miRNA-30a alleviates cerebral ischemic injury through enhancing beclin 1-mediated autophagy. Neurochem Res. 2014; 39: 1279-91. https://doi. org/10.1007/s11064-014-1310-6.

40. Zheng T, Lu Y. SIRT1 protects human lens epithelial cells against oxidative stress by inhibiting p53-dependent apoptosis. Curr Eye Res. 2016; 41: 1068-75. https://doi.org /10.3109/02713683.2015.1093641.

41. Tanaka H, Arakawa H, Yamaguchi T, Shiraishi K, Fukuda S, Matsui K, Takei Y, Nakamura Y. A ribonucleotide reductase gene involved in a p53-dependent cell-cycle checkpoint for DNA damage. Nature. 2000; 404: 42-9. https://doi. org/10.1038/35003506.

42. Marino G, Niso-Santano M, Baehrecke EH, Kroemer G. Self-consumption: the interplay of autophagy and apoptosis. Nat Rev Mol Cell Biol. 2014; 15: 81-94. https://doi. org/10.1038/nrm3735.

43. Gao W, Shen Z, Shang L, Wang X. Upregulation of human autophagy-initiation kinase ULK1 by tumor suppressor p53 contributes to DNA-damage-induced cell death. Cell Death Differ. 2011; 18: 1598-607. https://doi.org/10.1038/ cdd.2011.33.

44. Livesey KM, Kang R, Vernon P, Buchser W, Loughran P, Watkins SC, Zhang L, Manfredi JJ, Zeh HJ 3rd, Li L, Lotze MT, Tang D. p53/HMGB1 complexes regulate autophagy and apoptosis. Cancer Res. 2012; 72: 1996-2005. https:// doi.org/10.1158/0008-5472.CAN-11-2291.

45. Lee IH, Kawai Y, Fergusson MM, Rovira, II, Bishop AJ, Motoyama N, Cao L, Finkel T. Atg7 modulates p53 activity to regulate cell cycle and survival during metabolic stress. Science. 2012; 336: 225-8. https://doi.org/10.1126/ science. 1218395.

46. Xie ZZ, Li MM, Deng PF, Wang S, Wang L, Lu XP, Hu LB, Chen Z, Jie HY, Wang YF, Liu XX, Liu Z. Paris saponininduced autophagy promotes breast cancer cell apoptosis via the Akt/mTOR signaling pathway. Chem Biol Interact. 2017; 264: 1-9. https://doi.org/10.1016/j.cbi.2017.01.004. 